\title{
Clinical significance of microRNA-320a and insulin- like growth factor-1 receptor in early-onset preeclampsia patients
}

\section{Guilian Liao ( $\square$ guilian0601@163.com )}

Maternal and Child Health Hospital of Longgang District

\section{Danling Chen}

Maternal and Child Health Hospital of Longgang Distirct

Juan Li

Maternal and Child Health Hospital of Longgang District

Shaona Hu

Maternal and Child Health Hospital of Longgang District

\section{Research}

Keywords: Early-onset preeclampsia, microRNA-320a, Insulin-like growth factor-1 receptor, Diagnostic efficacy, Serum creatinine, Mean arterial pressure, Receiver operating characteristic

Posted Date: September 28th, 2020

DOl: https://doi.org/10.21203/rs.3.rs-81437/v1

License: (9) (1) This work is licensed under a Creative Commons Attribution 4.0 International License. Read Full License

Version of Record: A version of this preprint was published at European Journal of Obstetrics \& Gynecology and Reproductive Biology on August 1st, 2021. See the published version at https://doi.org/10.1016/j.ejogrb.2021.06.032. 


\section{Abstract \\ Background}

Currently, there is no reliable method to effectively predict and diagnose early-onset preeclampsia (EOPE). microRNAs (miRs) are powerful and promising biomarkers in multiple diseases, including EOPE. Therefore, the present study investigated the role of miR-320a in EOPE.

\section{Methods}

Expressions of miR-320a and insulin-like growth factor-1 receptor (IGF-1R) in serum of EOPE patients and normal pregnant women were detected. The clinical diagnostic efficacy of miR-320a and IGF-1R for EOPE was analyzed using receiver operating characteristic curve. The correlation between miR-320a expression and EOPE clinical indicators [mean arterial pressure (MAP), 24-h urinary protein excretion, serum creatinine (SCR), uric acid (UA), albumin (ALB) and platelet count] was analyzed. The correlation and binding relationship between miR-320a and IGF-1R was predicted and verified.

\section{Results}

miR-320a was upregulated, and IGF-1R was downregulated in EOPE patients with their differential expressions more obvious in severe EOPE than mild EOPE. miR-320a and IGF-1R possessed potent clinical diagnostic efficacy for EOPE. miR-320a expression showed a positive correlation with MAP, 24-h urinary protein excretion, UA and SCR levels, and a negative correlation with ALB level and platelet count in EOPE patients. Moreover, miR-320a targeted IGF-1R.

\section{Conclusion}

We demonstrated that miR-320a was aberrantly elevated in EOPE and showed powerful clinical diagnostic efficacy for EOPE, which may be achieved by directly targeting IGF-1R. This study provided great reference values for EOPE early diagnosis and novel targets for EOPE treatment.

\section{Introduction}

Preeclampsia (PE), a multisystem pregnancy syndrome, causes serious impacts on 2-8\% pregnant women globally [1]. PE pathophysiology is frequently initiated in the early pregnancy, while its clinical features usually show presence in mid-to-late pregnancy [2]. It's often manifested as new-onset hypertension and proteinuria, which can rapidly develop into severe complications and even lead to the death of mother and fetus [3]. Moreover, even if the fetus is born successfully, women with PE and their children are more likely to suffer from multiple metabolic diseases and cardiovascular complications in later life [4]. PE can be subcategorized as early-onset PE (EOPE, < 34-week gestation) and late-onset PE [5, 
6]. Compared with late-onset PE, EOPE possesses higher incidence rates for mothers and fetuses [7]. Key pathogenesis mechanisms of EOPE are widely accepted as insufficient trophoblast invasion in spiral arteries, changes in placental blood flow, and impairment of placental angiogenesis, resulting in restricted fetal growth [6]. Unfortunately, despite the development of medicine today, pharmacological agents for effectively treating EOPE are still lacking [8]. Furthermore, the widely used method to predict EOPE risk is based on the demographic characteristics and medical history of mothers, which has a poor predictive effect on EOPE and fails to identify patient-specific hazards [9]. Therefore, probing reliable and accurate biomarkers for EOPE is of great value for EOPE early prediction and effective prevention.

microRNAs (miRs), a group of single-stranded non-coding small molecules, regulate up to $50 \%$ protein coding gene expressions in mammals and participate in almost all cellular processes [10]. miRs are widely expressed in reproductive tissues, and miRs deregulation has been proven to be involved in placental formation and pregnancy-related pathological processes [11]. Furthermore, numerous studies have provided evidence that miRs exert effects on EOPE progression [12,13]. A previous study has pointed out that miR-320a shows a regulatory role in trophoblast cell behaviors [14]. However, the specific role of miR-320a in EOPE remains elusive. Therefore, the current study took the initiative to speculate that miR-320a may play a potential role in EOPE prediction and diagnosis through possible mechanisms. Consequently, we performed a series of molecular experiments to identify the underlying value of miR$320 \mathrm{a}$ in EOPE, with the purpose to provide some novel therapies against EOPE.

\section{Materials And Methods}

\section{Ethics statement}

The study got approval from the Ethics Committee of Maternal and Child Health Hospital of Longgang District. All patients have signed written informed consents.

\section{Sample collection}

A total of 70 cases of EOPE pregnant women (<34-week gestation) who were treated in the Department of Obstetrics and Gynecology of Maternal and Child Health Hospital of Longgang District from January 2019 to January 2020 were selected. Based on the 2002 American Academy of Obstetrics and Gynecology Practice Bulletin [15], the diagnostic criteria for EOPE were: (1) first detected hypertension after 20-week gestation (systolic blood pressure $\geq 140 \mathrm{~mm} \mathrm{Hg}$ or diastolic blood pressure $\geq 140 \mathrm{~mm} \mathrm{Hg}$ ); (2) urinary protein $>0.3 \mathrm{~g} / 24 \mathrm{~h}$ or random urinary protein $>1+$; (3) new-onset hypertension or one or more of adverse characteristics [including thrombocytopenia $(<100000 / \mu \mathrm{L})$, renal injury (creatinine $>$ $1.1 \mathrm{mg} / \mathrm{dL}$ ), pulmonary edema, liver dysfunction, cerebral or visual impairment]. In this study, 33 patients showing systolic blood pressure $\geq 160 \mathrm{~mm} \mathrm{Hg}$ or diastolic blood pressure $\geq 110 \mathrm{~mm} \mathrm{Hg}$ accompanied with mild proteinuria, or mild hypertension with severe proteinuria $(>2 \mathrm{~g} / 24 \mathrm{~h}$ or random urinary protein $>$ $2+$ ) were included in the severe EOPE group, and the rest (37 patients) were included in the mild EOPE group. In addition, 35 normal pregnant women in the same period were selected as the control group. All cases were singleton pregnancy without other maternal complications, such as multiple pregnancy, fetal 
abnormalities, premature rupture of membranes, maternal infection, and previous history of hypertension, diabetes, and heart and kidney diseases. The clinical baseline characteristics of the included subjects are shown in Table 1. The statistical indexes among the three groups showed no significant difference and were comparable (all $p>0.05$ ). The serum samples were collected from the cubital vein blood of pregnant women under fasting conditions on the first morning of admission, and centrifuged $\left(2000 \mathrm{~g}, 4^{\circ} \mathrm{C}\right)$ for $10 \mathrm{~min}$. The supernatant was transferred to the eppendorf tube for storage at $-80^{\circ} \mathrm{C}$. The sample was not subjected to any freeze-thaw cycles prior to this study.

Table 1

Clinical baseline characteristics of the included subjects

\begin{tabular}{|llll|}
\hline Variable & Control & Mild EOPE & Severe EOPE \\
\hline Cases & 35 & 37 & 33 \\
\hline Age (year) & $28.2 \pm 2.9$ & $27.9 \pm 3.1$ & $28.6 \pm 2.2$ \\
\hline Gestational weeks & $38.5 \pm 0.6$ & $38.2 \pm 1.2$ & $37.8 \pm 1.6$ \\
\hline
\end{tabular}

\section{Detection of clinicopathological characteristics}

The 24-h urinary protein excretion was measured using the biuret method. Mean arterial pressure (MAP) was measured using an ambulatory blood pressure monitor (ambulatory blood pressure system 3000 , Meigaoyi Medical Equipment Co., Ltd., Beijing, China). Serum creatinine (SCR), blood urea nitrogen (BUN), uric acid (UA) and albumin (ALB) levels and platelet count were detected using an 2700 automatic biochemical analyzer (Olympus Optical Co., Ltd., Tokyo, Japan).

\section{Reverse transcription quantitative polymerase chain reaction (RT-qPCR)}

Total RNA was extracted using TRIzol regent (Invitrogen Inc., Carlsbad, CA, USA) and then transcribed into cDNA using PrimeScript RT reagent kit (Takara Biotechnology Co., Ltd., Dalian, China). Then qPCR was performed using SYBR ${ }^{\circledR}$ Premix Ex Taq ${ }^{\text {TM }}$ II (Takara) on an ABI 7900HT fast PCR real-time system (Applied Biosystems, Foster City, CA, USA). The reaction conditions were 10-min pre-denaturation at $95^{\circ} \mathrm{C}$, and 40 cycles of 10 -s denaturation at $95^{\circ} \mathrm{C}, 20$-s annealing at $60^{\circ} \mathrm{C}$ and 34 -s extending at $72{ }^{\circ} \mathrm{C}$. U6 served as the internal control. Data were analyzed using the $2^{-\Delta \Delta C t}$ method. The primer sequences of miR-320a and U6 were synthesized by Sangon Biotech Co., Ltd. (Shanghai, China) (Table 2). 
Table 2

Primer sequences of miR-320a and U6

\begin{tabular}{|ll|}
\hline Gene & Primer \\
\hline hsa-miR-320a & F: 5'-CGGGGAGAGGGCGAAA-3' \\
\cline { 2 - 3 } & R: 5'-AGTGCAGGGTCCGAGGTATT-3' \\
\hline U6 & F: 5'-ATTGGAACGATACAGAGAAGATT-3' \\
\cline { 2 - 2 } & R: 5'-GGAACGCTTCACGAATTTC-3' \\
\cline { 2 - 2 } \\
\hline
\end{tabular}

\section{Enzyme-linked immunosorbent assay (ELISA)}

ELISA kit (R\&D Systems, Minneapolis, MN, USA) was used to detect insulin-like growth factor-1 receptor (IGF-1R) content in serum. Each well of coated enzyme-labeled plate was added with $10 \mu \mathrm{L}$ serum sample and $40 \mu \mathrm{L}$ sample diluent, which then underwent a 30 -min warm bath at $37^{\circ} \mathrm{C}$ after plate sealing. After 5 washes of the reaction plate, $50 \mu \mathrm{L}$ enzyme-labeled reagent was added, and then the plate was sealed and put in a $37^{\circ} \mathrm{C}$ warm bath for $30 \mathrm{~min}$. Next, the reaction plate was washed, and developing reagents $\mathrm{A}$ and $B\left(50 \mu \mathrm{L}\right.$, respectively) were added and mixed evenly for a 15 -min visualization at $37^{\circ} \mathrm{C}$. Afterwards, $50 \mu \mathrm{L}$ termination fluid was added to each well. Meanwhile, the color changed from blue to yellow. Finally, the optical density (OD) at $450 \mathrm{~nm}$ was determined using an Multiskan ${ }^{\mathrm{TM}} \mathrm{FC}$ microplate reader (Thermo Fisher Scientific Inc., Waltham, MA, USA). Serum IGF-1R level was calculated using the standard curve.

\section{Dual-luciferase reporter gene assay}

Through Starbase (http://starbase.sysu.edu.cn/index.php) [16], it was predicted that there were binding sites between miR-320a and IGF-1R. The complementary binding sequences and the mutant sequences of miR-320a and IGF-1R were amplified and cloned into pmiR-GLO luciferase vectors (Promega Corp., Madison, WI, USA) to construct the wild-type (WT) plasmid IGF-1R-WT and the corresponding mutant plasmid IGF-1R-MUT. As per the instructions of Lipofectaminetm 2000 (Invitrogen), IGF-1R-WT and IGF1R-MUT were co-transfected with mimic negative control (NC) or miR-320a mimic respectively into HEK293T cells (Shanghai Institute of Cellular Biology of Chinese Academy of Sciences, Shanghai, China). Luciferase activity was detected $48 \mathrm{~h}$ later.

\section{Statistical analysis}

SPSS 21.0 (IBM Corp., Armonk, NY, USA) and GraphPad Prism 8 (GraphPad Software, La Jolla, CA, USA) were used for data analysis and plotting. Kolmogorov-Smirnov test verified the data were in normal distribution. Measurement data were represented as mean \pm standard deviation. Pearson correlation coefficient method was used for the correlation analysis of serum miR-320a expression with IGF-1R expression and clinical indexes. The diagnostic value of miR-320a and IGF-1R for EOPE was analyzed using receiver operating characteristic (ROC) curve. The $\chi^{2}$ test was used for statistical analysis of classified data. $p<0.05$ indicated statistical significance. 


\section{Results}

\section{Comparison of clinicopathological characteristics between EOPE patients and normal pregnant women}

As shown by the comparison results of clinicopathological characteristics of 35 normal pregnant women and EOPE patients (37 mild EOPE and 33 severe EOPE), there were significant differences in MAP, 24-h urinary protein excretion, SCR, UA and ALB levels and platelet count, as well as the incidence of edema (Table 3) (all $p<0.05$ ). BUN and maternal body weight showed no remarkable difference (Table 3) (all $p>$ 0.05). Specifically, EOPE (mild/severe) patients had notably increased MAP level, 24-h urinary protein excretion and edema incidence relative to normal pregnant women, and severe EOPE patients showed higher levels of these indexes than mild EOPE patients. SCR and UA levels in severe EOPE patients were dramatically higher than those in normal pregnant women and mild EOPE patients, while showing no obvious difference between normal pregnant women and mild EOPE patients. Moreover, ALB level in EOPE patients was markedly reduced compared with normal pregnant women, and it was lower in severe EOPE patients than that in mild EOPE patients. Severe EOPE patients showed obviously decreased platelet counts and maternal body weight as compared to mild EOPE patients and normal pregnant women, while no noticeable difference was found between mild EOPE patients and normal pregnant women.

Table 3

Comparison of clinicopathological characteristics between EOPE patients and normal pregnant women

\begin{tabular}{|llll|}
\hline Variable & Control $(\mathbf{n}=35)$ & Mild EOPE $(\mathbf{n}=\mathbf{3 7})$ & Severe EOPE $(\mathbf{n}=\mathbf{3 3})$ \\
\hline MAP $(\mathrm{mm} \mathrm{Hg})$ & $92.37 \pm 8.13$ & $114.62 \pm 9.86^{\mathrm{a}}$ & $133.28 \pm 10.74^{\mathrm{ab}}$ \\
\hline Urinary protein $(\mathrm{g} / 24 \mathrm{~h})$ & - & $1.3 \pm 0.3$ & $2.5 \pm 0.3^{\mathrm{b}}$ \\
\hline $\mathrm{SCR}(\mu \mathrm{mol} / \mathrm{L})$ & $60.86 \pm 4.16$ & $63.23 \pm 3.82$ & $73.23 \pm 6.88^{\mathrm{b}}$ \\
\hline $\mathrm{BUN}(\mathrm{mmol} / \mathrm{L})$ & $4.55 \pm 0.85$ & $4.78 \pm 0.79$ & $4.96 \pm 0.56$ \\
\hline UA $(\mu \mathrm{mol} / \mathrm{L})$ & $252.64 \pm 39.73$ & $270.23 \pm 30.12$ & $397.92 \pm 39.02^{\mathrm{b}}$ \\
\hline ALB $(\mathrm{g} / \mathrm{L})$ & $39.74 \pm 2.65$ & $36.21 \pm 2.23^{\mathrm{a}}$ & $32.76 \pm 2.29^{\mathrm{ab}}$ \\
\hline Platelet $\left(\cdot 10^{9}\right)$ & $209.18 \pm 72.79$ & $187.57 \pm 53.14$ & $99.82 \pm 21.09^{\mathrm{b}}$ \\
\hline Maternal body weight $(\mathrm{kg})$ & $72.89 \pm 8.72$ & $72.47 \pm 8.59$ & $69.08 \pm 6.35^{\mathrm{b}}$ \\
\hline Edema & $0(0.00)$ & $26(44.07)$ & $39(95.12)^{\mathrm{b}}$ \\
\hline
\end{tabular}

miR-320a was highly expressed in serum of EOPE patients and had high clinical diagnostic efficacy for EOPE 
miR-320a expression in serum of EOPE (mild/severe) patients and normal pregnant women was detected. It was found that the miR-320a expression in EOPE was remarkably increased, and severe EOPE patients $(t=6.937, p<0.0001)$ had noticeably higher miR-320a expression than mild EOPE patients $(\mathrm{t}=$ $3.528, p<0.01$ ) (Fig. 1A). miR-320a clinical diagnostic value for EOPE was analyzed using ROC curve, and the ROC curve (area under ROC curve $=0.790 ; 71.40 \%$ specificity; $70.00 \%$ sensitivity) (Fig. 1B) $(p<$ 0.001 ) revealed that serum miR-320a expression had high diagnostic value for EOPE.

\section{miR-320a was closely correlated with clinical indexes in EOPE patients}

As presented by Table 3, clinical indexes such as MAP, 24-h urinary protein excretion, SCR, UA, ALB and platelet count in EOPE patients showed remarkable differences. To further study the relationship between miR-320a expression and EOPE, we analyzed the correlation between miR-320a expression and these abnormal clinical indexes. In this correlation analysis, mild and severe EOPE patients were combined analyzed. According to our results, serum miR-320a expression was moderately positively correlated with MAP (Fig. 2A) $(r=0.515, p<0.0001)$, 24-h urinary protein excretion (Fig. 2B) $(r=0.533, p<0.0001)$, SCR (Fig. 2C) $(r=0.499, p<0.0001)$ and UA (Fig. 2D) $(r=0.511, p<0.0001)$ levels. miR-320a expression also showed a moderate negative correlation with ALB level (Fig. 2E) $(r=-0.509, p<0.0001)$ and platelet count (Fig. 2F) $(r=-0.535, p<0.0001)$.

\section{miR-320a targeted IGF-1R expression in EOPE patients}

As has been evidenced, IGF-1R is involved in placental development and may be associated with EOPE [17]. Through Starbase (http://starbase.sysu.edu.cn/index.php), we predicted that miR-320a can target IGF-1R. In addition, pan-cancer analysis of hsa-miR-320a and IGF-1R expression in 32 cancer samples showed that hsa-miR-320a expression is negatively correlated with IGF-1R in most cases (Fig. 3A). Based on the above database prediction and existing research results, IGF-1R expression in EOPE was detected. We found that it was dramatically downregulated in EOPE patients, and severe EOPE patients $(\mathrm{t}=8.159$, $p<0.0001)$ showed markedly lower IGF-1R expression than mild EOPE patients $(\mathrm{t}=3.660, p<0.01)$ (Fig. 3B). Moreover, the correlation of miR-320a with IGF-1R was analyzed (mild and severe EOPE were combined assessed). As shown by our findings, miR-320a expression was negatively correlated with IGF1R (Fig. 3C) $(r=-0.335, p<0.01)$. Furthermore, a dual-luciferase reporter gene assay was used to verify the targeted relationship between miR-320a and IGF-1R. The luciferase activity of HEK293T cells cotransfected with PGL-IGF-1R-WT and miR-320a mimic was noticeably decreased (Fig. 3D) $(p<0.05)$, which was indicative of the targeted binding relationship between miR-320a and IGF-1R. From all above, we confirmed that there was a negative correlation between miR-320a and IGF-1R in serum of EOPE patients, and miR-320a may play a role in EOPE via directly targeting IGF-1R.

\section{Serum IGF-1R expression had clinical diagnostic efficacy for EOPE}


To further study the clinical significance of serum IGF-1R expression in EOPE, we analyzed the diagnostic efficacy of IGF-1R for EOPE using ROC curve. The area under ROC curve was 0.811 , the specificity was $71.40 \%$, and the sensitivity was $85.70 \%$ (Fig. 4$)(p<0.001$ ), which showed that IGF-1R possessed good diagnostic efficacy for EOPE and had certain reference value for EOPE early diagnosis.

\section{Discussion}

EOPE severely threatens maternal, perinatal and neonatal survival [18]. miRs have been demonstrated to be crucial regulators in the pathogenesis of pregnancy-related diseases [19]. The present study revealed that miR-320a was aberrantly elevated in EOPE and showed powerful clinical diagnostic efficacy for EOPE, which may be achieved by directly targeting IGF-1R.

It has shown that deregulated miRs in placental tissues in pregnant woman are tightly implicated in EOPE, showing a potential to serve as powerful diagnostic indicators for EOPE [20]. As has been evidenced, miR-320a regulates trophoblast cell proliferation, invasion and apoptosis; changes of trophoblast cell behaviors are frequently reported to be associated with pregnancy diseases [14, 21]. According to our findings, miR-320a expression in serum of EOPE patients was remarkably increased, and it was noticeably higher in severe EOPE patients than mild EOPE patients. We also identified that miR320a had potent clinical diagnostic value for EOPE using ROC curve, as confirmed by an area under ROC curve of 0.790 , a specificity of $71.40 \%$ and a sensitivity of $70.00 \%$. In support of these, a previous study has provided evidence that miR-320a is abnormally enhanced in PE patients, promising to be a therapeutic target for PE therapy [22]. It is well established that increased urinary protein excretion, MAP, UA and SCR levels during pregnancy are potent indexes for EOPE prediction [23-26]. In addition, decreased platelet count and ALB level also show a predictive and diagnostic value for EOPE $[27,28]$. Therefore, the correlation between miR-320a expression and these clinical indexes was detected. We found that miR-320a expression was moderately positively correlated with MAP, 24-h urinary protein excretion, SCR and UA levels, while negatively correlated with ALB level and platelet count. Consistently, a prior work has proven that noticeably elevated miR-320a expression shows a close correlation with PE progression [29]. Taken together, we confirmed that miR-320a was implicated in EOPE with its abnormal upregulation showing potent clinical predictive and diagnostic value for EOPE.

Subsequently, we shifted to investigating the downstream mechanism of miR-320a in EOPE. A previous study has proposed that IGF-1R shows a close relation with placental development and fetal growth [17]. In the current study, through a series of predictions and experiments, we identified IGF-1R as the target gene of miR-320a; IGF-1R expression was dramatically downregulated in EOPE, and severe EOPE patients showed markedly lower IGF-1R expression than mild EOPE patients. Likewise, a number of studies have demonstrated the targeted regulatory relationship between miR-320a and IGF-1R in multiple diseases [30-32]. Furthermore, a previous work has provided compelling evidence that IGF-1R plays a crucial role in PE via the involvement of trophoblast syncytialization [33]. Nevertheless, the interplay between miR$320 \mathrm{a}$ and IGF-1R in EOPE is not fully understood, which, on the other hand, demonstrates the novelty of 
this study. Briefly, we proved that there was a negative correlation between miR-320a and IGF-1R expression in EOPE patients, and miR-320a exert effects on EOPE via directly targeting IGF-1R.

Next, the diagnostic efficacy of IGF-1R for EOPE was further analyzed. According to the results shown by ROC curve ( 0.811 areas under ROC curve, $71.40 \%$ specificity and $85.70 \%$ sensitivity), IGF-1R was of a great value for EOPE early diagnosis. As has been proven previously, IGF-1R variant is valuable in the prediction of breast cancer in PE women [34]. Placental IGF-1R numbers are positively related to placental weight [35]. From all above, this study for the first time provided evidence for the function of IGF-1R in direct prediction and diagnosis of EOPE.

All in all, this study found that miR-320a was abnormally elevated and had potent clinical predictive and diagnostic efficacy for EOPE, which may be mediated by targeting IGF-1R in EOPE. Our results provided a certain reference value for EOPE early diagnosis. miR-320a and IGF-1R may develop as possible novel targets for EOPE treatment. According to the reported studies at home and abroad, we speculated that miR-320 may regulate cellular insulin resistance and then play a role in EOPE via targeting IGF-1R and inhibiting the PI3K/Akt pathway. In the future, we will explore this point and study the regulatory mechanism of miR-320a and IGF-1R in EOPE from the perspective of epigenetics.

\section{Declarations}

\section{Consent for publication}

Not applicable.

\section{Availability of data and materials}

All the data generated or analyzed during this study are included in this published article.

\section{Competing Interests}

The authors declare that they have no competing interests.

\section{Acknowledgements}

Not applicable.

\section{Funding}

Not applicable.

\section{Patient consent for publication}

Not applicable

\section{Author Contributions}


LGL is the guarantor of integrity of the entire study; LGL contributed to the study concepts, study design, and definition of intellectual content, SNH contributed to the literature research, LGL contributed to the manuscript preparation,editing and review; LGL contributed to the clinical studies; DLC and JL contributed to the experimental studies and data acquisition; DLC contributed to the data analysis and statistical analysis. All authors read and approved the final manuscript.

\section{References}

1. Ornaghi S, Paidas MJ. Novel Therapy for the Treatment of Early-Onset Preeclampsia. Clin Obstet Gynecol. 2017;60:169-82.

2. Barnea ER, Vialard F, Moindjie H, Ornaghi S, Dieudonne MN, Paidas MJ. Prelmplantation Factor (PIF*) endogenously prevents preeclampsia: Promotes trophoblast invasion and reduces oxidative stress. J Reprod Immunol. 2016;114:58-64.

3. Rana S, Lemoine E, Granger JP, Karumanchi SA. Preeclampsia: Pathophysiology, Challenges, and Perspectives. Circ Res. 2019;124:1094-112.

4. Malik A, Jee B, Gupta SK. Preeclampsia. Disease biology and burden, its management strategies with reference to India. Pregnancy Hypertens. 2019;15:23-31.

5. Burton GJ, Redman CW, Roberts JM, Moffett A. Pre-eclampsia: pathophysiology and clinical implications. BMJ. 2019;366:I2381.

6. Raymond D, Peterson E. A critical review of early-onset and late-onset preeclampsia. Obstet Gynecol Surv. 2011;66:497-506.

7. Farladansky-Gershnabel S, Gal H, Kidron D, Krizhanovsky V, Amiel A, Sukenik-Halevy R, et al. Telomere Homeostasis and Senescence Markers Are Differently Expressed in Placentas From Pregnancies With Early- Versus Late-Onset Preeclampsia. Reprod Sci. 2019;26:1203-9.

8. Ahmed A, Rezai H, Broadway-Stringer S. Evidence-Based Revised View of the Pathophysiology of Preeclampsia. Adv Exp Med Biol. 2017;956:355-74.

9. Wright $\mathrm{D}$, Wright A, Nicolaides $\mathrm{KH}$. The competing risk approach for prediction of preeclampsia. Am J Obstet Gynecol. 2020;223:12-23. e17.

10. Bidarimath M, Khalaj K, Wessels JM, Tayade C. MicroRNAs, immune cells and pregnancy. Cell Mol Immunol. 2014;11:538-47.

11. Morales Prieto DM, Markert UR. MicroRNAs in pregnancy. J Reprod Immunol. 2011;88:106-11.

12. Lv Y, Lu C, Ji X, Miao Z, Long W, Ding H, et al. Roles of microRNAs in preeclampsia. J Cell Physiol. 2019;234:1052-61.

13. Xu P, Zhao Y, Liu M, Wang Y, Wang H, Li YX, et al. Variations of microRNAs in human placentas and plasma from preeclamptic pregnancy. Hypertension. 2014;63:1276-84.

14. Liu RH, Meng Q, Shi YP, Xu HS. Regulatory role of microRNA-320a in the proliferation, migration, invasion, and apoptosis of trophoblasts and endothelial cells by targeting estrogen-related receptor gamma. J Cell Physiol. 2018;234:682-91. 
15. Bulletins-Obstetrics ACoP. ACOG practice bulletin. Diagnosis and management of preeclampsia and eclampsia. Number 33, January 2002. Obstet Gynecol. 2002;99:159-67.

16. Li JH, Liu S, Zhou H, Qu LH, Yang JH. starBase v2.0: decoding miRNA-ceRNA, miRNA-ncRNA and protein-RNA interaction networks from large-scale CLIP-Seq data. Nucleic Acids Res. 2014;42:D92-7.

17. Takai H, Kondoh E, Mogami H, Kawasaki K, Chigusa Y, Sato M, et al. Placental sonic hedgehog pathway regulates foetal growth via insulin-like growth factor axis in preeclampsia. J Clin Endocrinol Metab 2019.

18. Song J, Li Y, An RF. Identification of Early-Onset Preeclampsia-Related Genes and MicroRNAs by Bioinformatics Approaches. Reprod Sci. 2015;22:954-63.

19. Lycoudi A, Mavreli D, Mavrou A, Papantoniou N, Kolialexi A. miRNAs in pregnancy-related complications. Expert Rev Mol Diagn. 2015;15:999-1010.

20. Lykoudi A, Kolialexi A, Lambrou GI, Braoudaki M, Siristatidis C, Papaioanou GK, et al. Dysregulated placental microRNAs in Early and Late onset Preeclampsia. Placenta. 2018;61:24-32.

21. Huppertz B. Traditional and New Routes of Trophoblast Invasion and Their Implications for Pregnancy Diseases. Int J Mol Sci 2019; 21.

22. Zhong Y, Zhu F, Ding Y. Differential microRNA expression profile in the plasma of preeclampsia and normal pregnancies. Exp Ther Med. 2019;18:826-32.

23. Majak GB, Reisaeter AV, Zucknick M, Lorentzen B, Vangen S, Henriksen T, et al. Preeclampsia in kidney transplanted women; Outcomes and a simple prognostic risk score system. PLoS One. 2017;12:e0173420.

24. Nobakht MGBF. Application of metabolomics to preeclampsia diagnosis. Syst Biol Reprod Med. 2018;64:324-39.

25. Ryu A, Cho NJ, Kim YS, Lee EY. Predictive value of serum uric acid levels for adverse perinatal outcomes in preeclampsia. Medicine. 2019;98:e15462.

26. Zhu XL, Wang J, Jiang RZ, Teng YC. Pulsatility index in combination with biomarkers or mean arterial pressure for the prediction of pre-eclampsia: Systematic literature review and meta-analysis. Ann Med. 2015;47:414-22.

27. Dai DM, Cao J, Yang HM, Sun HM, Su Y, Chen YY, et al. Hematocrit and plasma albumin levels difference may be a potential biomarker to discriminate preeclampsia and eclampsia in patients with hypertensive disorders of pregnancy. Clin Chim Acta. 2017;464:218-22.

28. Mayer-Pickel K, Stern C, Eberhard K, Lang U, Cervar-Zivkovic M. Changes of platelet count throughout pregnancy in women with antiphospholipid syndrome. J Reprod Immunol. 2019;136:102612.

29. Gao T, Deng M, Wang Q. MiRNA-320a inhibits trophoblast cell invasion by targeting estrogen-related receptor-gamma. J Obstet Gynaecol Res. 2018;44:756-63.

30. Li C, Zhang S, Qiu T, Wang Y, Ricketts DM, Qi C. Upregulation of long non-coding RNA NNT-AS1 promotes osteosarcoma progression by inhibiting the tumor suppressive miR-320a. Cancer Biol Ther. 2019;20:413-22. 
31. Shu S, Liu X, Xu M, Gao X, Chen S, Zhang L, et al. MicroRNA-320a acts as a tumor suppressor in endometrial carcinoma by targeting IGF-1R. Int J Mol Med. 2019;43:1505-12.

32. Wang J, Shi C, Wang J, Cao L, Zhong L, Wang D. MicroRNA-320a is downregulated in non-small cell lung cancer and suppresses tumor cell growth and invasion by directly targeting insulin-like growth factor 1 receptor. Oncol Lett. 2017;13:3247-52.

33. Zhou Z, Zhang Q, Lu X, Wang R, Wang H, Wang YL, et al. The proprotein convertase furin is required for trophoblast syncytialization. Cell Death Dis. 2013;4:e593.

34. Powell MJ, Von Behren J, Neuhausen S, Reynolds P, Benz CC. Functional IGF1R variant predicts breast cancer risk in women with preeclampsia in California Teachers Study. Cancer Causes Control. 2017;28:1027-32.

35. Diaz E, Cardenas M, Ariza AC, Larrea F, Halhali A. Placental insulin and insulin-like growth factor I receptors in normal and preeclamptic pregnancies. Clin Biochem. 2005;38:243-7.

\section{Figures}
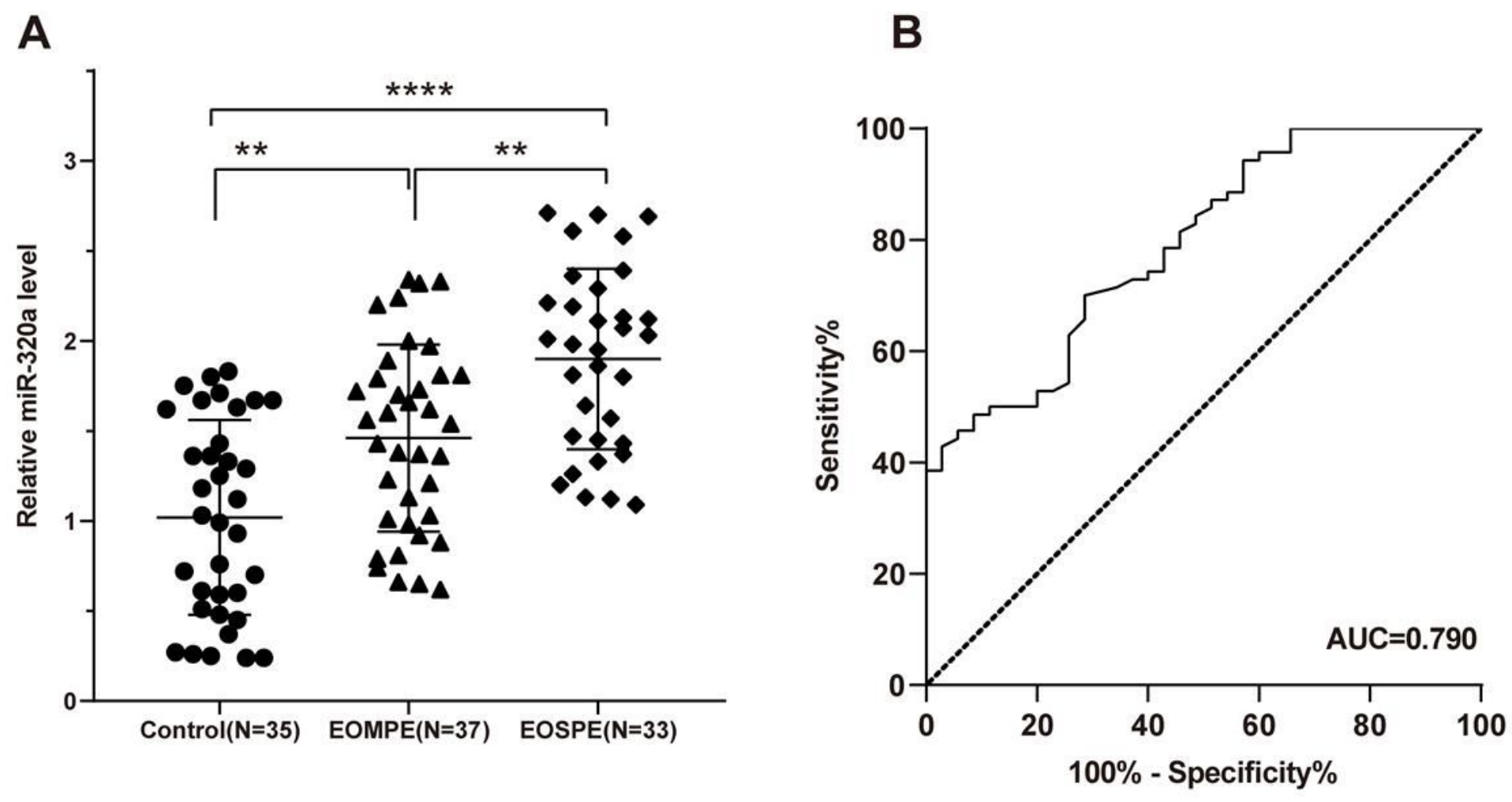

Figure 1

miR-320a is highly expressed in serum of EOPE patients and has high clinical diagnostic efficacy. A. The expression of miR-320a in serum of EOPE patients and normal pregnant women was detected using RTqPCR; B. miR-320a clinical diagnostic value for EOPE was analyzed using ROC curve; the area under the 
ROC curve was 0.790 , the specificity was $71.40 \%$, and the sensitivity was $70.00 \%$.** $<<0.01$, *** $<$ 0.0001 .

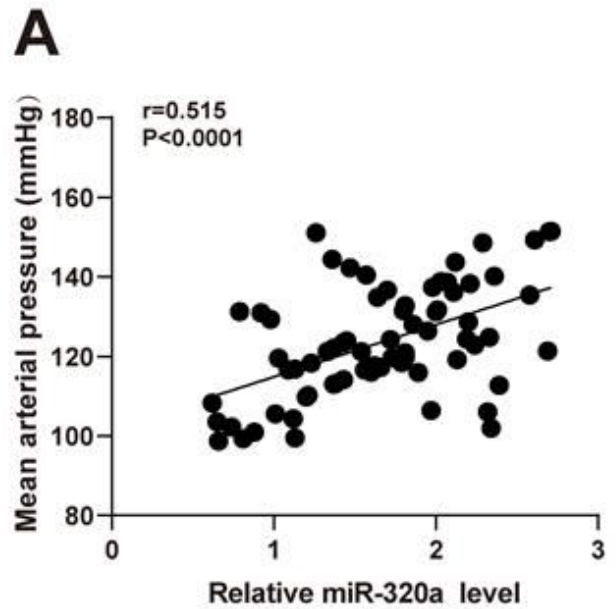

D

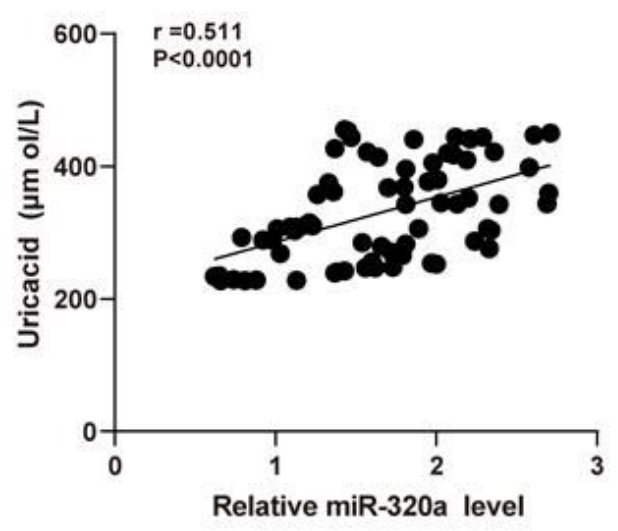

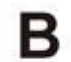

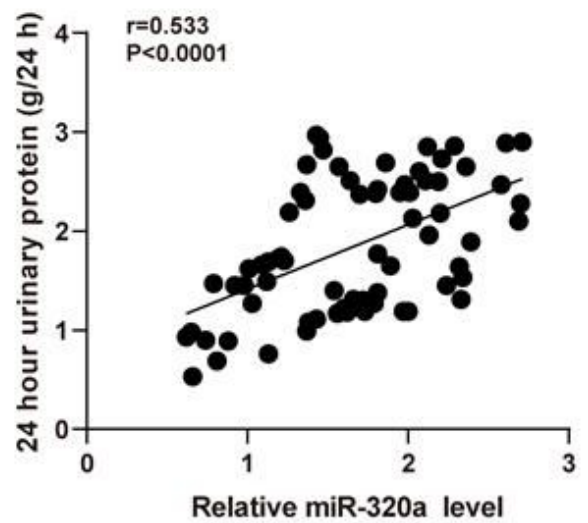

E

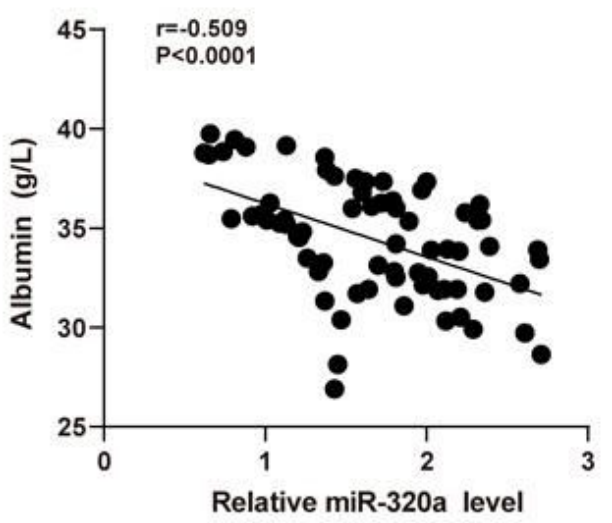

C

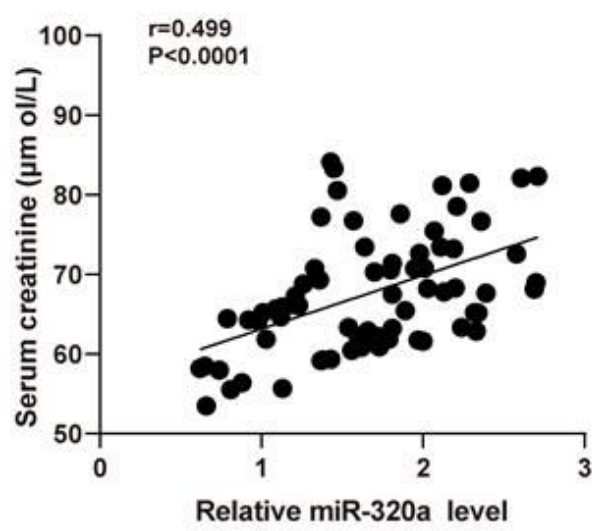

$\mathbf{F}$

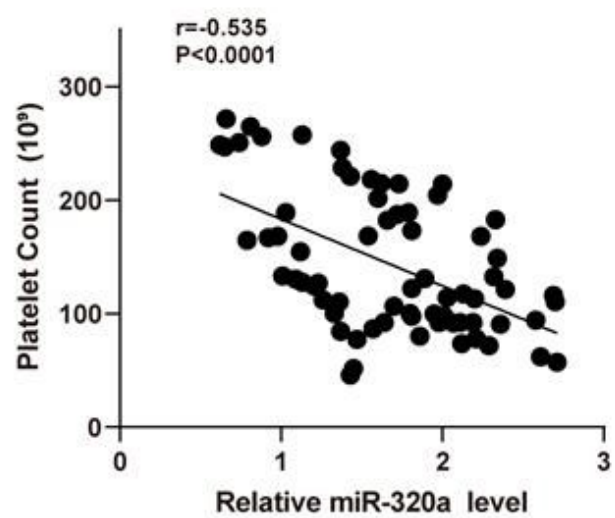

Figure 2

miR-320a is closely correlated with clinical indexes in EOPE patients. A-D. Pearson analysis showed that miR-320a was moderately positively correlated with MAP, 24-h urinary protein excretion, SCR and UA levels in EOPE patients; E/F. Pearson analysis showed that miR-320a was moderately negatively correlated with ALB level and platelet count in EOPE patients. 
A

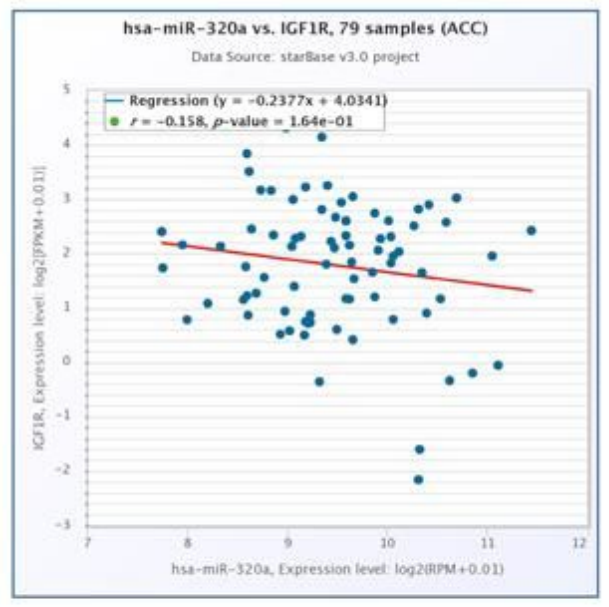

C

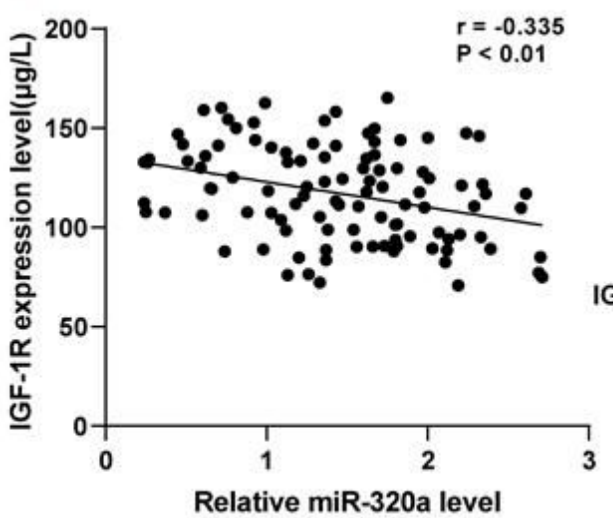

B
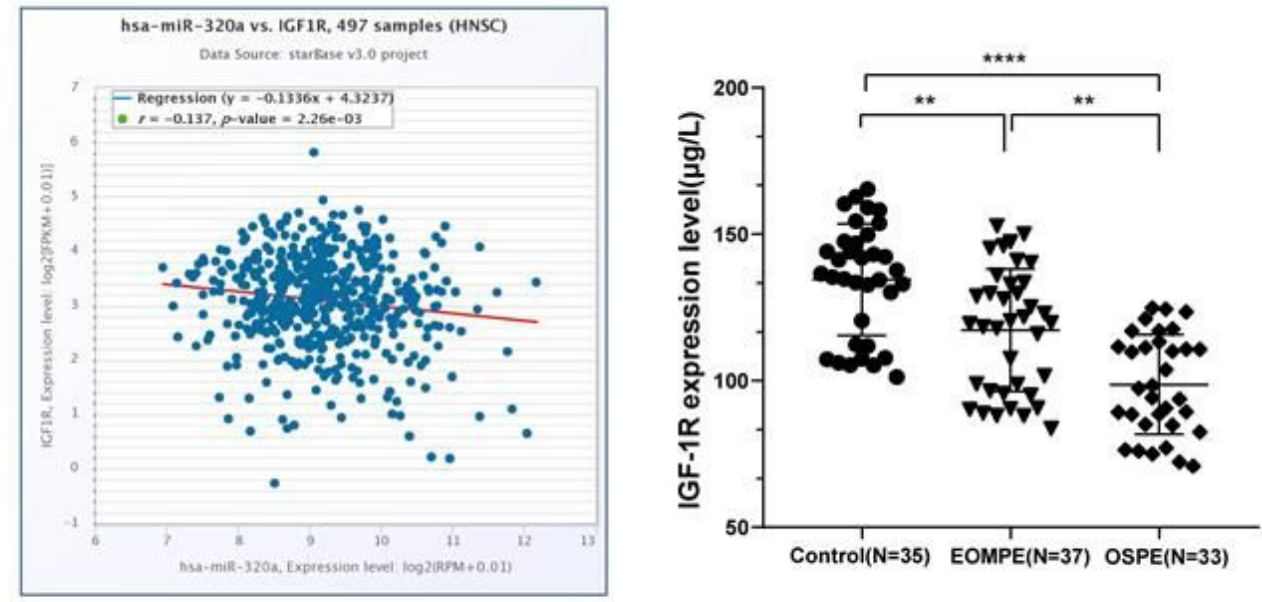

D

IGF-1R 5' cacCUGUUUACUAGCUAGCUUUa 3'<smiles>C=CC(C)C(C)C(C)C(C)C(C)C(C)C(C)C(C)C=C1C=CC=C1</smiles>
miR-320a 3' agcGGGAGA -GUUGGGUCGAAAa 5' IGF-1R-MUT 5' cacGUGAGAAGUUGGUUCGAAAa 3'

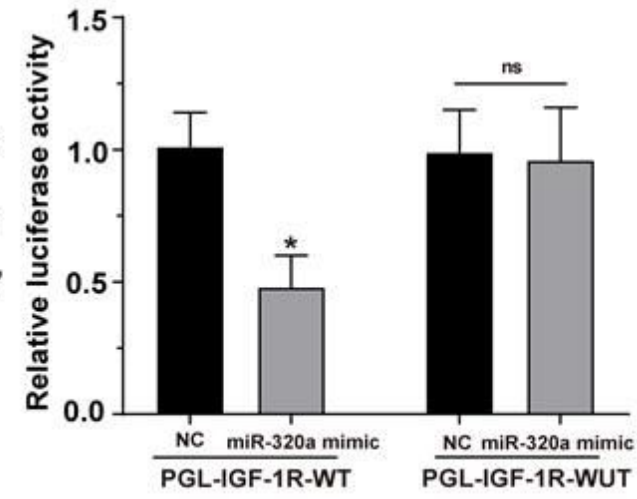

Figure 3

miR-320a targets IGF-1R expression in EOPE patients. A. Results of pan-cancer analysis (adrenocortical carcinoma and head and neck squamous cell carcinoma) through Starbase database (http://starbase.sysu.edu.cn/index.php): hsa-miR-320a expression was negatively correlated with IGF-1R expression; B. IGF-1R expression in serum of EOPE patients and normal pregnant women was detected using ELISA; C. The correlation between miR-320a and IGF-1R serum expression was analyzed using Pearson analysis; D. Dual-luciferase reporter gene assay was used to verify the targeted relationship between miR-320a and IGF-1R. ${ }^{*} p<0.05,{ }^{*} p<0.01$, ${ }^{\star \star \star *} p<0.0001$, ns $p>0.05$. 


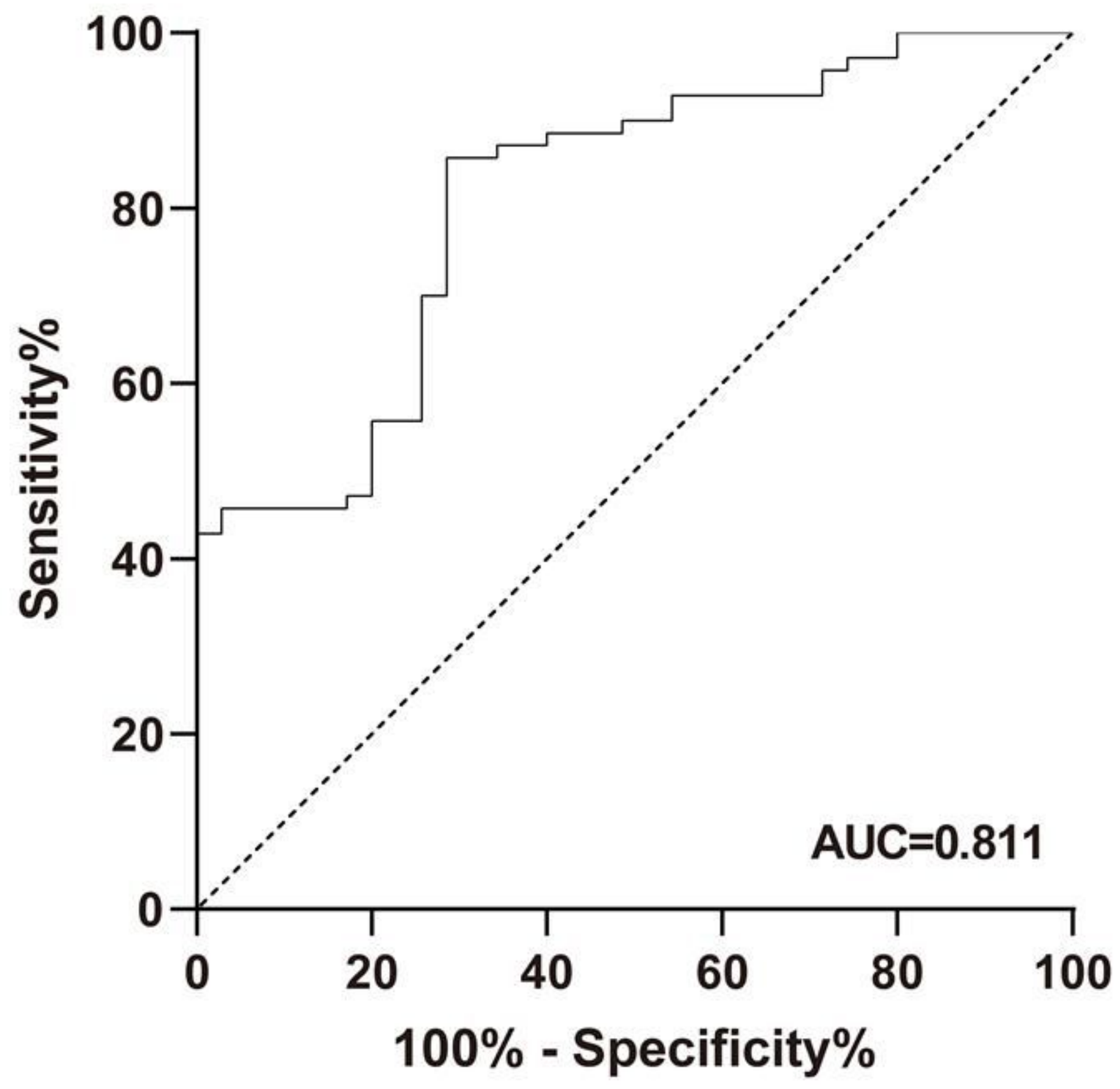

Figure 4

Serum IGF-1R expression has clinical diagnostic efficacy for EOPE. The diagnostic efficacy of IGF-1R for EOPE was analyzed using ROC curve; the area under ROC curve was 0.811 , the specificity was $71.40 \%$, and the sensitivity was $85.70 \%$. 